\title{
Étude comparative de la croissance de Clarias gariepinus et Clarias ngamensis en élevage semi- intensif de la ferme Mwema a Lubumbashi.
}

Ipungu Lushimba. *, Kayuma Makila*, Labe Nkum Nziel. *, Malangu Binemo. *, Binemo Kanyama. *, Ompey Ishwishwom. *, Ilunga Nkulu Bulundu. *, Numbi Malale. *, Kidinda Mako **, Kapemba Katangu***., Ngoy Kiayima*.

*Faculté de médecine vétérinaire université de Lubumbashi, Service de Pisciculture et pathologie des poissons, B.P 1825, Lubumbashi, RDC.

**Faculté de santé publique, université de Kabinda, RDC

***Institut supérieur d'étude agro vétérinaire, Sandoa, RDC

Service de pisciculture et pathologie des poissons. Fac de médecine vétérinaire de l'université de Lubumbashi : b.p.1825 Rd Congo Email de l'auteur correspondant:drpaulinmakila@gmail.com; raymondipungu@gmail.com

Mots-clés : croissance, Clarias gariepinus, Clarias ngamensis, élevage, Lubumbashi.

Keywords: growth, Clarias gariepinus, Clarias ngamensis, breeding, Lubumbashi

Publication date 31/07/2019, http://www.m.elewa.org/JAPS

\section{RESUME}

La comparaison de la croissance de Clarias gariepinus et de Clarias ngamensis dans un système semi-intensif basé sur le poids et la taille lors de la reproduction artificielle à la ferme Mwema à Lubumbashi. L'étude a portée sur un lot homogène de 100 alevins dont 50 de Clarias gariepinus ayant un poids initial de $30 \mathrm{~g}$ et $22 \mathrm{~cm}$ de longueur corporelle, en suite 50 de Clarias ngamensis dont le poids initial était de $30 \mathrm{~g}$ et $20 \mathrm{~cm}$ de longueur corporelle. Au bout de 5 mois d'étude, nous avions obtenu un poids moyen de $82 \mathrm{~g}$ et $29 \mathrm{~cm}$ pour Clarias gariepinus et $60 \mathrm{~g}$ et $25 \mathrm{~cm}$ pour Clarias ngamensis. En faisant recours à la méthode d'échantillonnage, aux paramètres physico-chimiques de l'eau, aux paramètres mesures et a la reproduction induite, les résultats obtenus montrent qu'il y a différence significative entre le poids moyen de Clarias et de la taille. Il ressort que la plaque vomérienne que possède le Clarias gariepinus a une influence sur la valorisation des aliments mis à sa disposition par rapport au Clarias ngamensis élevé dans l'étang.

\section{SUMMARY}

The comparison of the growth of Clarias gariepinus and Clarias ngamensis in a semiintensive system based on weight and size during artificial propagation on the Mwema farm in Lubumbashi. The study was carried out on a homogeneous batch of 100 fry including 50 Clarias gariepinus with an initial weight of $30 \mathrm{~g}$ and $22 \mathrm{~cm}$ of body length, followed by Clarias ngamensis whose initial weight was $30 \mathrm{~g}$ and $20 \mathrm{~cm}$ of body length. After 5 months of study, we obtained an average weight of $82 \mathrm{~g}$ and $29 \mathrm{~cm}$ for Clarias gariepinus and $60 \mathrm{~g}$ and $25 \mathrm{~cm}$ for Clarias ngamensis. Using the sampling method, the physicochemical parameters of the water, the measured parameters and the induced reproduction, the results obtained show that there is a significant difference between the average weight of Clarias and the size - It appears that the vomereal plate that Clarias gariepinus possesses has an influence on the valorization of the food put at its disposal compared to the Clarias ngamensis raised in the pond. 


\section{INTRODUCTION}

La pêche est une importante source d'aliment pour l'humanité. Elle assure, en outre, un emploi et procure des bénéfices économiques à ceux qui la pratiquent. Elle est une activité régulatrice de l'économie de plusieurs pays et source de diversification alimentaire en matière de protéines animales car le poisson demeure la protéine la plus accessible et la moins couteuse pour les populations. Source importante de nutriment, vitamines et minéraux, les poissons pris uniquement avec certains produits végétaux constitue un aliment complet (AMAKOE, 2011). La République Démocratique du Congo, traverse une période de crise ou la majorité de sa population est pauvre. Buté a ce problème, l'élevage de Clarias gariepinus et de Clarias ngamensis offre des possibilités énormes dans la production des poissons, est le moyen plus facile de fournir les protéines à d'origine animale dont la teneur en celle-ci est à peu près la même que celle de la viande (Fontaine Et Bailp, 2004). La croissance est un processus biologique complexe qui fait intervenir des nombreux facteurs (Kestemont, 2003). Le Clarias ngamensis a un corps allongé et

\section{MATERIEL ET METHODES}

3.1 Milieu : Nos recherches ont eu lieu à la ferme Mwema de Janvier à Mai 2014. La ferme Mwema est une exploitation agropastorale située à $25 \mathrm{Km}$ de la ville de Lubumbashi, à $25^{\circ} \mathrm{C}$ comme température moyenne annuelle, à une attitude moyenne de plus ou moins $1.230 \mathrm{~m}$. Le sol est variable mais à prédominance sablo argileux de couleur rouge. Végétation est couverte d'une savane boisée.

3.2 Animaux : Notre étude a porté sur 20 alevins homogènes de Clarias gariepinus et Clarias ngamensis provenant de poisson-chat de l'INERA KIPOPO, dont le poids moyen était de $30 \mathrm{~g}$ et $22 \mathrm{~cm}$ pour Clarias gariepinus, puis $30 \mathrm{~g}$ et $20 \mathrm{~cm}$ pour Clarias ngamensis. Ces poissons ont été élevés dans un système semi-intensif au site précité pendant une période allant du mois de Janvier au mois de Mai 2014.

3.3 Matériel : Pour la réalisation de cette étude, nous avions utilisé à part le matériel cylindrique, à longue nageoires dorsales et anales, quelque peu anguilliforme. En général, sans nageoire adipeuse, à tête aplatie et hautement massifiée sans écaille et couverte des mucus. La bouche entourée de quatre paires des barbillons. Les nageoires pectorales sont armés d'épines non venimeuses fortement développés servant à la locomotion hors de l'eau et à la protection sous les opercules se trouve cinq arcs branchiaux (Teygels, 1996). Le Clarias gariepinus est une espèce tolérante au large spectre des températures, à des niveaux de salinités élevés, des faibles concentrations en oxygène dissous dans l'eau aussi les eaux turbides et la forte densité, ce qui explique son aire de reproduction relativement grande (Hechtt, 1982 ; Hengs Awat et al, 1997). Le poisson-chat Africain, particulièrement le Clarias gariepinus et le Clarias ngamensis vivent et grandissent dans une large gamme de température $\left(20\right.$ à $\left.30^{\circ} \mathrm{C}\right)$. ils sont capables, grâce à un organe de respiration complémentaire qui leur permet d'utiliser l'oxygène de l'air atmosphérique et rejeter l'air viciée à la surface de l'eau (Viveen et al, 1985 ; Melard, 2002).

biologique qui est le Clarias, aussi le matériel non biologique notamment :

- Le thermomètre aquatique: pour prélever la température de l'eau de l'étang

- Une balance de précision : pour la pesée des alevins

- Le mètre ruban : pour mesurer la taille des alevins

- L'épuisette en plastique: pour faire le tri des alevins

- Le filet : pour capturer les alevins,

- Les bassins en plastique : pour recueillir les alevins prélevés de l'étang,

- Le Bic : pour la prise des données

- Les papiers: pour écrire les données de prélèvement

- L'étang : lieu de vie des alevins

- Les seaux: pour recueillir les alevins prélevés dans l'étang 
- Les couteaux, pinces, seringues, sécateurs, un mortier + pilon, sérum physiologique, aliment et les papyrus.

Selon notre dispositif expérimental, nous avions utilisé 2 étangs dans lesquels avions reparti 50 alevins par étang, de même poids de sorte que chacun puisse subir un traitement équivalent de l'autre.

\subsection{METHODES}

3.4.1 Méthode d'échantillonnage: Les poissons ont été échantillonnés mensuellement par diverses techniques de pêche et du matériel pouvant nous permettre la prise en compte du poids et de la taille. Nous avons utilisé les filets maillants de 10, $12,15,20 \mathrm{~mm}$ des petites épuisettes, des grosses épuisettes, les bacs en plastique, le mètre ruban et la balance de précision.

\subsubsection{Paramètres physico-chimiques de l'eau dans l'étang}

3.4.2.1 prélèvements de la température : $\mathrm{La}$ température est l'élément physique très important gouvernant les activités du poisson. Elle était prélevée à l'aide d'un thermomètre aquatique.

Tableau 1 : Résumé des températures de l'eau $\left({ }^{\circ} \mathrm{C}\right)$ et le délai d'incubation des œufs/heure

\begin{tabular}{|c|c|}
\hline Température de l'eau en $\left({ }^{\circ} \mathbf{C}\right)$ & Délai d'incubation des œufs $/$ heure \\
\hline 20 & $57 \mathrm{~h} 00^{\prime}$ \\
21 & $46 \mathrm{~h} 00^{\prime}$ \\
22 & $38 \mathrm{~h} 00^{\prime}$ \\
23 & $23 \mathrm{~h} 00^{\prime}$ \\
24 & $29 \mathrm{~h} 00^{\prime}$ \\
24 & $27 \mathrm{~h} 00^{\prime}$ \\
26 & $25 \mathrm{~h} 00^{\prime}$ \\
27 & $23 \mathrm{~h} 00^{\prime}$ \\
28 & $22 \mathrm{~h} 00^{\prime}$ \\
29 & $21 \mathrm{~h} 00^{\prime}$ \\
30 & $20 \mathrm{~h} 00^{\prime}$ \\
\hline
\end{tabular}

L'examen du tableau 1 démontre que le développement des larves et rapide et s'accélère avec l'augmentation de la température de l'eau, plus température est élevée, plus sera l'éclosion rapide (Defreyene, 2005)

3.4.2.2 prélèvements du potentiel d'hydrogène : Le $\mathrm{pH}$ a été mesuré à l'aide d'un $\mathrm{pH}$-mètre ATC/HANNA de précision de 2.1 la transparence de l'eau a été mesuré en $\mathrm{cm}$ au moyen d'un disque de Secchi muni d'un bâton gradué qui a servi à la prise de la disparition des cadrans des disques.

3.4.2.3 Prélèvement de l'oxygène dissous : L'oxygène dissous été mesuré à l'aide d'un Oxythermomètre (oxyure en $\mathrm{mg} / \mathrm{l}$ ).

3.4.3 Paramètre mesures de poids et de longueur: Le poids était déterminé à l'aide d'une balance de précision en vue de calculer les différents paramètres de croissance. La taille était déterminée respectivement à l'aide d'un mètre ruban en vue de calculer la longueur corporelle du poisson.

3.4.4 Alimentation des géniteurs : La mise en condition optimale de reproduction des géniteurs a nécessité une période pré- expérimentale de 2 mois au cours desquels on devrait vérifier l'adaptation des poissons à l'aliment et les paramètres physicochimiques de l'eau.

3.4.5 Dispositif expérimental : Les larves ont été déplacées dans des bassines contenant 12 litres d'eau pour une mise en charge de 4.17 larves par litre soit 50 larves par bassine.

4 Productions des larves et expérimentation

4.1 Production des larves : La production des larves était réalisée en écloserie apres la méthode utilisée et celle proposée par Viveen et al. (1985) et reprise par Degraaf et al. (1995).

4.2 Expérimentation : L'expérimentation était conduite de la manière suivante :

D'abord à la résorption de la vésicule vitelline, les larves ont été nourries aux zooplanctons pendant 10 jours puis les juvéniles ont été placés dans les bassines contenant 12 litres d'eau et avaient reçus les aliments secs, qui étaient distribués trois fois par jour : le matin, à midi et le soir. Après 10 jours de sevrage, les juvéniles furent soumis à la deuxième phase d'alimentation de 30 jours d'élevage. La fréquence de distribution d'aliment était de 3 fois par jour à raison de 10\% de la biomasse par jour. 
Tableau 2 : distribution de Clarias ngamensis en poids et taille

\begin{tabular}{|l|l|l|l|l|l|}
\hline Espèces & mois & $\begin{array}{l}\text { Poids moyen } \\
\text { (gr) }\end{array}$ & Taille $\mathbf{( c m )}$ & $\begin{array}{l}\text { Ecart-type } \\
\text { (poids) }\end{array}$ & $\begin{array}{l}\text { Ecart-type } \\
\text { (taille) }\end{array}$ \\
\hline \multirow{4}{*}{ Clarias ngamensis } & premier & $\mathbf{3 0}$ & $\mathbf{1 8 . 3}$ & $\mathbf{3 0 \pm 0 \mathrm { g }}$ & $18.3 \pm 0 \mathrm{~cm}$. \\
\cline { 2 - 6 } & deuxième & $\mathbf{3 5}$ & 19 & $35 \pm 2 \mathrm{~g}$. & de $19 \pm 0 \mathrm{Cm}$. \\
\cline { 2 - 6 } & troisième & $\mathbf{4 1}$ & 23 & $41 \pm 1 \mathrm{~g}$ & $23 \pm 1 \mathrm{~cm}$. \\
\cline { 2 - 6 } & quatrième & 50 & 24 & $50 \pm 4 \mathrm{~g}$. & $24 \pm 2 \mathrm{~cm}$. \\
\cline { 2 - 6 } & cinquième & 60 & $\mathbf{2 5}$ & $60 \pm 1 \mathrm{~g}$. & $25 \pm 2 \mathrm{Cm}$ \\
\hline
\end{tabular}

Tableau 3 : distribution de Clarias gariepinus poids et taille

\begin{tabular}{|l|l|l|l|l|l|}
\hline Espèces & mois & $\begin{array}{l}\text { Poids } \\
\text { moyen } \mathbf{( g r})\end{array}$ & Taille $\mathbf{( c m )}$ & $\begin{array}{l}\text { Ecart-type } \\
\text { (poids) }\end{array}$ & $\begin{array}{l}\text { Ecart-type } \\
\text { (taille) }\end{array}$ \\
\hline \multirow{4}{*}{ Clarias gariepinus } & premier & 35 & 21 & $35 \pm 0 \mathrm{~g}$. & $21 \pm 0 \mathrm{Cm}$ \\
\cline { 2 - 6 } & deuxième & 43 & $\mathbf{2 2}$ & $43 \pm 2 \mathrm{~g}$. & $22 \pm 0 \mathrm{Cm}$ \\
\cline { 2 - 6 } & troisième & $\mathbf{4 9}$ & $\mathbf{2 4}$ & $49 \pm 4 \mathrm{~g}$. & $24 \pm 4 \mathrm{Cm}$ \\
\cline { 2 - 6 } & quatrième & $\mathbf{6 3}$ & $\mathbf{2 6}$ & $63 \pm 3 \mathrm{~g}$. & $26 \pm 2 \mathrm{Cm}$ \\
\cline { 2 - 6 } & cinquième & $82 \mathrm{~g}$ & 29 & $82 \pm 1 \mathrm{~g}$. & $29 \pm 1 \mathrm{Cm}$ \\
\hline
\end{tabular}

\section{$5 \quad$ DISCUSSION DES RESULTATS}

Durant notre expérimentation dans l'étude comparative de la croissance des Clarias gariepinus et de Clarias ngamensis, il ressort que plusieurs paramètres sont envisagés à savoir : la température, l'alimentation, le $\mathrm{pH}$, le taux de nitrate, la turbidité, la croissance est surtout influencée par l'alimentation et l'environnement des poissons dans les étangs. Ceci entraine le plus souvent une compétition alimentaire et un ralentissement de la croissance des individus. Comparativement aux résultats trouvés par un bon nombre des chercheurs dans ce domaine, le Clarias gariepinus a montré une croissance plus élevée que le Clarias ngamensis. Il ressort de l'analyse des fréquences des tailles que la cohorte des Clarias gariepinus a fait une bonne croissance, ceci confirme bien les hypothèses de reproduction des poissons-chats durant une période de l'année Chikou, 2006. Notre travail corrobore avec Barras et Almedida, 2001, qui eux aussi ont trouvées que les Clarias gariepinus se situeraient entre 25 et $30 \mathrm{~cm}$ de la longueur totale durant une période de 6mois. Toutefois il existe des différences très marquées de la croissance individuelle et des individus des plus de $30 \mathrm{~cm}$ peuvent être rencontrées dans la population. Ainsi nous constatons que le rythme de croissance est très lent chez les Clarias ngamensis du fait de leur bourrelet des plaques vomériennes et se développe mieux dans les fleuves surtout dans les conditions naturelles (Prodepaak, 2009). Le Clarias gariepinus manifeste une croissance rapide du fait de leur plaque vomérienne et c'est un carnivore par excellence (MICHA, 1973).

\section{CONCLUSION GENERALE ET RECOMMANDATION}

Durant notre expérimentation, l'accent a porté sur l'étude comparative de Clarias gariepinus et Clarias ngamensis en fonction du poids et de la taille dans les étangs à la ferme Mwema pendant une période thermique allant du mois de janvier au mois de Mai 2014. Notre étude a portée sur un lot homogène de 100 alevins dont 50 de Clarias gariepinus et 50 autres des Clarias ngamensis. Au bout de 5 mois, nous avons obtenu le poids moyen de $82 \mathrm{~g}$ et $29 \mathrm{~cm}$ de longueur pour les Clarias gariepinus, puis $60 \mathrm{~g}$ et $25 \mathrm{~cm}$ pour les Clarias ngamensis.En faisant recours à la méthode d'échantillonnage, aux paramètres physicochimiques de l'eau, aux paramètres de mesure et à la reproduction induite, les résultats obtenus ont montré que les Clarias gariepinus accusent un poids plus considérable que le Clarias ngamensis. Ceci s'explique par le fait que le Clarias gariepinus présente des plaques vomériennes lui permettant de mieux valoriser des aliments à sa disposition par rapport aux Clarias ngamensis élevé dans l'étang. Étant donné que les plaques vomériennes du Clarias gariepinus lui permet de mieux valoriser les aliments mis à sa disposition, nous recommandons aux pisciculteurs d'élever les Clarias gariepinus vu ses performances pondérales en élevage. 


\section{BIBLIOGRAPHIE}

Amakoke. A 2011. Formation en pisciculture: production d'alevins et gestion de ferme piscicole, coordination togolaise des organisations paysannes et de producteurs agricoles. Lomé.

Bard et Coll, 1974.manuel de pisciculture tropicale, paris

Barras.E et Almedida, 2001. Dynamics of intracohort cannibalism in cultured fish; aquaculture research, 33:461-479.

Chikou.A, 2006 Etude de la démographie et de l'exploitation halieutique de six espèces de poissons-chats (Teleostei, Siluriformes) dans le delta de l'Ouémé au Bénin.Thèse de doctorat, université de liège, p459

Degraaf F.G.Ju et Janssen. H, 1995. Artificial reproduction and pond rearing of the Africa cat fish Clarias gariepinus in subsahara africa a hand book, F.A.O, fisheries technical paper Rome.

Defreyne.P.S, 2005. Utilisation de sous-produits agro-industriels dans l'alimentation des géniteurs du poisson-chat africain Clarias gariepinus (burchell, 1822) au Rwanda et sur le système reproducteur, mémoire, fac, université notre dame de la paix, 57p.

Donalson et Laune.Y, 1979. Aquaculture encyclopédie universel corpus 5, Namur.

Fontaine.P et Bailp.y, 2004. Contribution à l'étude des besoins nutritionnels chez les larves et juvéniles du clarias, 336p.

Hecht.T, 1982 (1). Intensives rearing of Clarias gariepinus larval (c.pisces). mobil rescachargnenium.

Hecht.T, 1986 (2). Intensives of Clarias gariepinus larval (piscis claridae). mobil research aquarium south africa.

Hecht.T et Britz.P.J, 1997. The culture of sharptooth catfish, Clarias gariepinus in southern Africa national scientific programmes report.

Janssen.J.A.L, 1987. Élevage du poisson chat africain clarias lazera (int et al, 1840) en république centre africaine alevinage en écloserie, FAO, Rome, document.

Kestermont.P, 2003. Nutrition et alimentation des poissons D.E.S, fac. Université notre dame de la paix, Namur.

Melard.C, 2002. Bases biologiques de l'aquaculture notes de cours, liège.
Micha J.C, 1973. First guide lims for the culture of Clarias lazera in central Africa, aquaculture center afrique.

Prodepaak, 2009. Reproduction induite du poissonchat africain Clarias gariepinus, manuel de vulgarisation, Lubumbashi. 